\title{
NUMERICAL SIMULATION OF THE FLOW AROUND A WIND WHEEL WITH ROTATING CYLINDRICAL BLADES
}

\author{
Tanasheva N.K. ${ }^{1}$, Bakhtybekova A.R. ${ }^{1 *}$, Sakipova S.E. ${ }^{1}$, Minkov L.L. ${ }^{2}$, \\ Shuyushbaeva N.N. ${ }^{3}$, Kasimov A.R. ${ }^{3}$ \\ 1E.A. Buketov Karaganda University, Karaganda, Kazakhstan, asem.alibekova@inbox.ru \\ 2 National Research Tomsk State University, Tomsk, Russia \\ ${ }^{3}$ Sh.Ualikhanov Kokshetau State University, Kokshetau, Kazakhstan
}

\begin{abstract}
The article discusses the results of numerical simulation of the flow around a wind wheel with blades in the form of rotating cylinders using the software package ANSYS. The advantage of a wind turbine with rotating cylindrical blades in comparison with traditional blade installations is the starting moment and the beginning of energy production at a wind speed of (2 - 3) $\mathrm{m} / \mathrm{s}$. A mathematical model has been developed based on threedimensional Navier-Stokes equations in a rotating system. The corresponding boundary conditions are formulated. A calculated pattern of the flow around the wind wheel with rotating cylindrical blade is obtained. There are shown regions of the velocity field with turbulent vortices, which are formed at high Reynolds numbers. The degree of influence of the angular speed of rotation of the wind wheel on the magnitude of the moment of forces at various speeds of the incoming air flow has been determined.
\end{abstract}

Keywords: wind wheel, ANSYS, rotating cylinder, mathematical model, moment of forces.

\section{Introduction}

Experimental research of a wind power plant is a rather time-consuming process, and requires certain climatic conditions with wind components. In such cases, it is advisable to use a theoretical study using mathematical modeling [1]. A wind power plant with rotating cylinders is distinguished by the Magnus effect involved in its operation from existing blade wind turbines. The Magnus force that occurs on a rotating cylinder due to the asymmetry separation of the flow from its surface can be an order of magnitude higher than the lifting force of the traditional blade. Based on this, in the case of the installation under consideration, we can expect an increase in the driving force of the wind wheel, as well as other operational advantages. One of the main advantages of a wind power plant with cylindrical blades is the start of energy generation at low wind speeds starting from $2 \mathrm{~m} / \mathrm{s}$, in contrast to the blades with $5 \mathrm{~m} / \mathrm{s}$ [2].

In the modern scientific world, numerical and theoretical studies of the aerodynamic characteristics of a mathematical model of a wind power plant with a rotating cylinder reach a qualitative level using the NavierStokes equations. This type of research is necessary to optimize the model configuration parameter [3, 4]. It should be noted that there are some difficulties in solving problems of the dynamics of continuous media, with the modeling of media due to the small values of the Mach number $(\mathrm{M}<0.1)$ [5-8]. To solve this problem, the ANSYS program was chosen as a CFD solver tool for the simulation procedure, since it provides flexible and accurate capabilities when working with non-stationary flow conditions, and also offers a wide range of turbulence models. In three-dimensional (3D) modeling, you can set real operating boundary conditions and measure the values of interest at any given location [9-13].

\section{The mathematical model of a wind wheel}

\subsection{System of equations}

In the simulation of rotation, the three-dimensional Navier-Stokes equations in a rotating frame of reference have the form:

continuity equations (conservation of mass)

$$
\frac{\partial \rho}{\partial t}+\nabla \cdot \rho \bar{v}_{r}=0
$$


conservation of momentum equation

$$
\nabla \cdot\left(\rho \bar{v}_{r} \cdot \bar{v}_{r}\right)+\rho\left(2 \bar{\omega} \times \bar{v}_{\tau}+\bar{\omega} \times \bar{\omega} \times \bar{r}\right)=-\nabla p+\nabla \bar{\tau}_{r},
$$

where $\rho$-air density; $\bar{v}_{r}-$ relative speed; $\bar{\omega}-$ angular velocity; $\bar{\omega} \times \bar{v}_{\tau}-$ coriolis acceleration; $\bar{\omega} \times \bar{\omega} \times \bar{r}-$ centripetal acceleration; $\bar{\tau}_{r}$ - viscous stress, $p$ - pressure.

Since the simulation of a rotating wind wheel involves non-stationary aerodynamic effects, choosing the right turbulence model is of great importance. The realizable $\mathrm{k}-\varepsilon$ turbulence model was used to model the currents around the rotating wind wheel. This model is used to model single-phase flows at high Reynolds numbers. The physical interface is suitable for incompressible flows, weakly compressible flows, and compressible flows at low M (Mach number), usually M less than 0.3).

The main assumptions made when describing the flow of air flowing around a wind power plant:

1. Due to the low values of $M$, when the ratio of the gas velocity to the local speed of sound $M<<0.1$, the motion of the air flow is described by the equations valid for an incompressible medium.

2. Due to the large $\operatorname{Re}$ (the ratio of the inertial forces to the viscosity forces, $\operatorname{Re}>10^{4}$ ), the flow is turbulent.

3. Due to the low values of $M$ and slight temperature changes in the vicinity of the wind wheel, the flow is isothermal.

The equations implemented at the k- $\varepsilon$ interface for solving turbulent flow problems include the NavierStokes equations (RANS) averaged by Reynolds for momentum conservation and the continuity equation for mass conservation (3). The flow near the walls is modeled using wall functions $f_{i}$.

$$
\rho \bar{u}_{j} \frac{\partial \bar{u}_{i}}{\partial x_{j}}=\rho \bar{f}_{i}+\frac{\partial}{\partial x_{j}}\left[-\bar{\rho} \delta_{i j}+\mu\left(\frac{\partial \bar{u}_{i}}{\partial x_{j}}+\frac{\partial \bar{u}_{j}}{\partial x_{i}}\right)-\rho \overline{u_{i}^{\prime} u_{j}^{\prime}}\right] \text {, }
$$

where $\rho \bar{f}_{i}-$ external forces; $\bar{\rho} \delta_{i j}-$ pressure forces; $\mu\left(\frac{\partial \bar{u}_{i}}{\partial x_{j}}+\frac{\partial \bar{u}_{j}}{\partial x_{i}}\right)-$ viscous forces; $\rho \overline{u_{i}^{\prime} u_{j}^{\prime}}-$ Reynolds stresses.

The left-hand side of this equation is the change in the average momentum of the liquid element due to the instability of the average flow and the convection of the average flow. This change is balanced by the averaged external forces, pressure forces, viscous forces, and apparent stress $-\rho \overline{u_{i}^{\prime} u_{j}^{\prime}}$ due to the fluctuating velocity field, commonly referred to as the Reynolds stress. Equations (4 - 6) are an implementation of the k$\varepsilon$ turbulence model:

$$
\begin{aligned}
& \frac{\partial}{\partial t}(\rho k)+\frac{\partial}{\partial x_{i}}\left(\rho k u_{i}\right)-\frac{\partial}{\partial x_{j}}\left[\left(\mu+\frac{\mu_{t}}{\sigma_{k}}\right) \frac{\partial k}{\partial x_{j}}\right]=G_{k}-\rho \varepsilon, \\
& \frac{\partial \rho \varepsilon}{\partial t}+\frac{\partial \rho \varepsilon u_{j}}{\partial x_{j}}=\frac{\partial}{\partial x_{j}}\left[\left(\mu+\frac{\mu_{t}}{\sigma_{\varepsilon}}\right) \frac{\partial \varepsilon}{\partial x_{j}}\right]+\rho \varepsilon\left(C_{1} S-C_{2} \frac{\varepsilon}{k+\sqrt{v \varepsilon}}\right), \\
& \mu_{t}=\rho C_{\mu} \frac{k^{2}}{\varepsilon},
\end{aligned}
$$

where $G_{k}$ - the turbulence kinetic energy of due to the average velocity gradients; $k$-is the turbulent kinetic energy; $\varepsilon$-turbulent dissipation; $\mu_{t}$-eddy viscosity; $\sigma_{k}, \sigma_{\varepsilon}, \mathrm{C}_{1}, \mathrm{C}_{2}-$ model constants $\left(\sigma_{k}=1.0, \sigma_{\varepsilon}=1.2\right.$, $\left.\mathrm{C}_{1}=1.44, \mathrm{C}_{2}=1.9\right) ; S=\sqrt{2 S_{i j} S_{i j}}-$ is the modulus of the strain rate tensor; $\mathrm{C}_{\mu}$ - dimensionless empirical constant.

\subsection{Boundary conditions and computational model}

To increase the computational efficiency, it was decided to use the symmetric and rotational nature of the wind turbine operation $[9,10]$. Figure 1 shows the computational domain and the built - in grid with boundary conditions. The boundary conditions are presented in Table 1. 


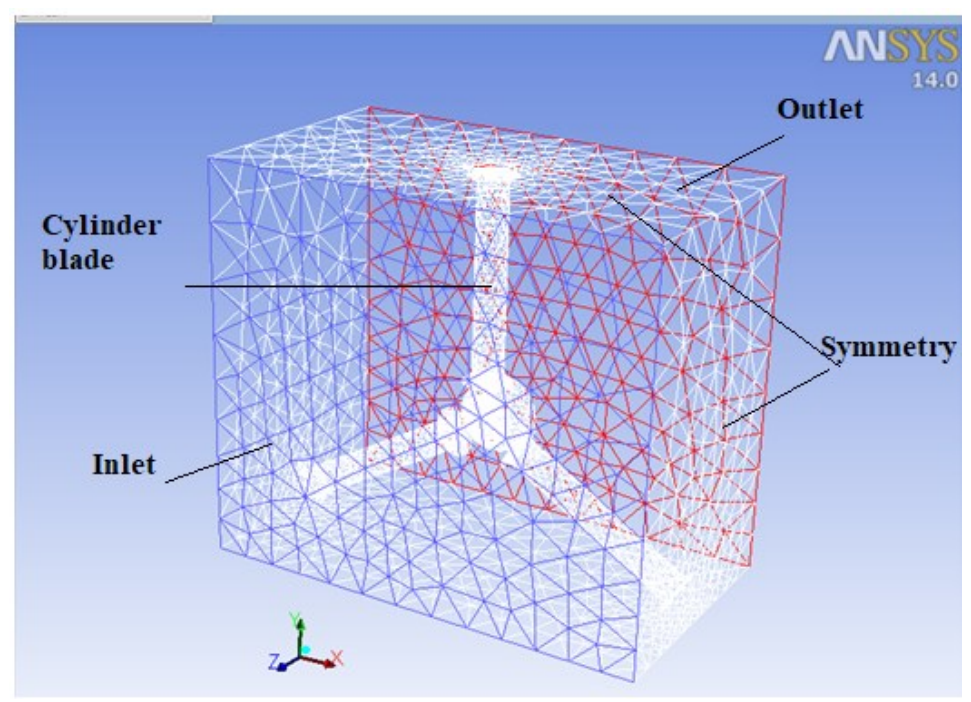

Fig.1. Computing area of the wind wheel

Table 1-List of boundary conditions

\begin{tabular}{|c|c|}
\hline \multicolumn{2}{|c|}{ Boundary conditions } \\
\hline \multicolumn{2}{|l|}{ Inlet } \\
\hline Type & Inlet speed \\
\hline Initial pressure gauge $(\mathrm{Pa})$ & 0 \\
\hline Air flow velocity, $\mathrm{m} / \mathrm{s}$ & $3,5,7,10,15$ \\
\hline Turbulence intensity (\%) & 5 \\
\hline Coefficient of turbulent viscosity & 10 \\
\hline \multicolumn{2}{|l|}{ Outlet } \\
\hline Type & Outlet pressure \\
\hline Pressure gauge $(\mathrm{Pa})$ & 0 \\
\hline Reverse flowof turbulent intensity (\%) & 5 \\
\hline Coefficient of backflow of turbulent intensity (\%) & 10 \\
\hline \multicolumn{2}{|l|}{ Blade surface } \\
\hline Type & Wall \\
\hline Shiftcondition & Noslipping \\
\hline \multicolumn{2}{|c|}{ Periodic conditions } \\
\hline Type & Rotation \\
\hline Wind wheel rotation speed (rpm) & $300,500,700$ \\
\hline
\end{tabular}

\section{Discussion of numerical simulation results}

As a result of numerical simulation, a picture of the air flow around the wind wheel was obtained (Figure 2). As can be seen from the figure, a three-dimensional vortex characteristic of a turbulent flow at high Reynolds numbers is observed in the rear part. This phenomenon is characterized by the constant distribution's formation of air flow characteristics's inconsistent pulsations in wavelength range from the minimum determined by the viscosity forces to the maximum determined by the flow conditions at the boundary. Studying the methods of numerical study of wind power plants, it was found that the result of modeling should be the dependences of mechanical parameters, namely:

1) The functional dependence of mechanical power on air flow parameters and speed:

$$
P_{m}=f\left(v, n_{1}\right) \text {, }
$$


where $P_{m}$ - mechanical output power; $v$-air flow rate; $n_{1}-$ the number of revolutions of the wind wheel.

2) The directly proportional dependence of the mechanical moment on the mechanical power:

$M_{m}=\frac{P_{m}}{\omega}$,

where $M_{m}$ - mechanical moment; $\omega$ - the angular speed of rotation of the propeller.

3) The equation describing the motion of the wind wheel:

$$
J \cdot \frac{d w}{d t}=M_{m}-M_{g}
$$

where $J$-wind wheel moment of inertia; $M_{g}$ - useful moment load on the shaft $[1,6]$.

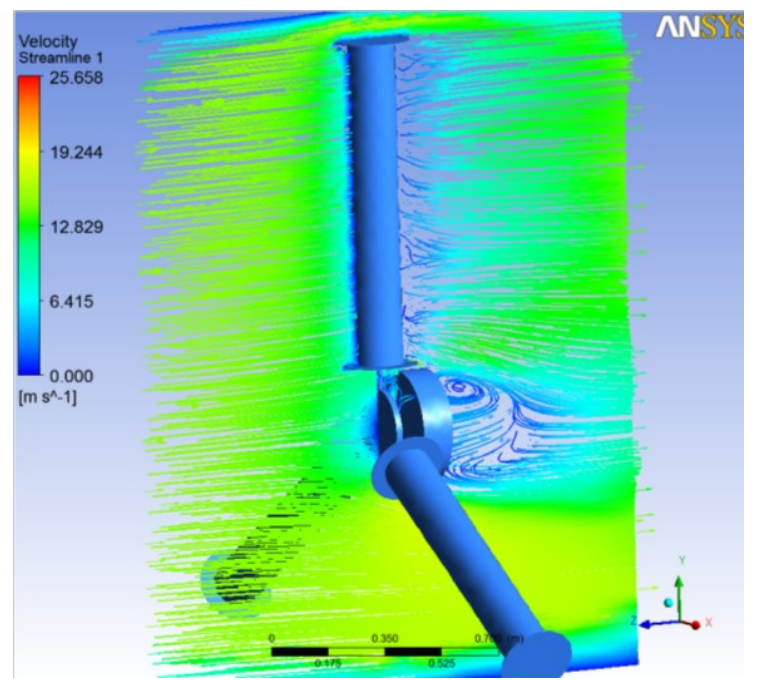

$a$

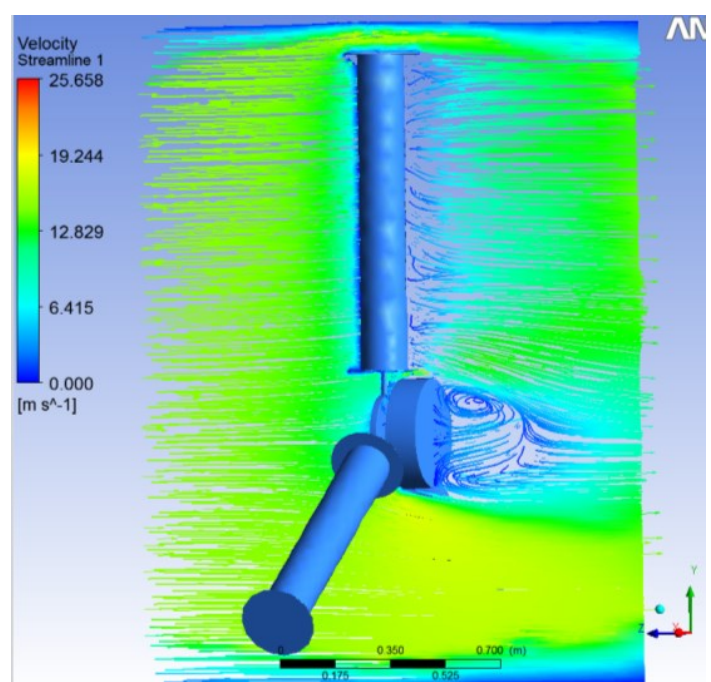

$b$

Fig.2. Pictures of the flow around the wind wheel velocity field at $\mathrm{v}=15 \mathrm{~m} / \mathrm{s}$ : a) front view; b) rear view.

The above dependencies show the complete dynamic picture of the wind wheel. Figure 3 shows influence diagram of the equivalent concentrated forces $Q_{v}, Q_{w}$ and the force $F_{g}$, which forms the moment of the payload on the propeller shaft.

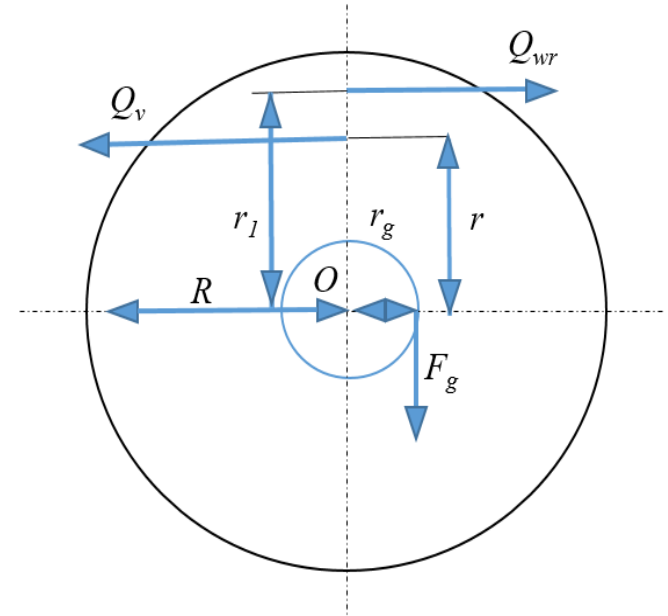

Fig.3. Scheme of action of the resulting equivalent forces on the wind wheel:

$\mathrm{R}$ - outer radius of the wind wheel; $\mathrm{r}_{\mathrm{g}}$ - force application shoulder $\mathrm{F}_{\mathrm{g}} ; \mathrm{O}$ - the axis of the wind wheel; $\mathrm{r}$ - force application shoulder $\mathrm{Q}_{\mathrm{v}} ; \mathrm{r}_{1}-$ force application shoulder $\mathrm{Q}_{\mathrm{wr}}$.

The moments of forces affecting the propeller, relative to the axis of rotation, is the product of the above forces by the length of the shoulder (Fig.). Let's define the moments value: 


$$
M_{v}=Q_{v} \cdot r
$$

- the moment made by the air flow with a speed v:

$$
M_{w r}=Q_{w r} \cdot r_{1}
$$

- the moment made by the payload of the wind turbine:

$$
M_{g}=F_{g} \cdot r_{g} \text {. }
$$

The total mechanical moment made by the blades of the propeller [1]:

$$
M_{m}=M_{v}-M_{w r}-M_{g}
$$

where $Q_{v}$ - force application shoulder r; $Q_{w r}$ - force application shoulder $\mathrm{r}_{1 ;} F_{g}$ - force application shoulder $\mathrm{r}_{\mathrm{g}}$.

Figures 4 (a-d) show the effect of the speed of rotation of the wind turbine $n_{1}$ on the moment of forces $\mathrm{M}$ acting on the wind turbine for different speeds of the incoming flow $\mathrm{v}$ and different speeds of rotation of the cylinders $\mathrm{n}$. According to the given dependencies, it is possible to determine the speed of free rotation of the wind wheel, i.e. without taking into account the moment of friction forces in the bearings and the moment of forces arising in the generator. The nature of the linear dependence of the values of the moment of forces on the speed of free rotation of the wind wheel does not contradict the results [1] $\mathrm{n}_{1}, \mathrm{rpm}$.

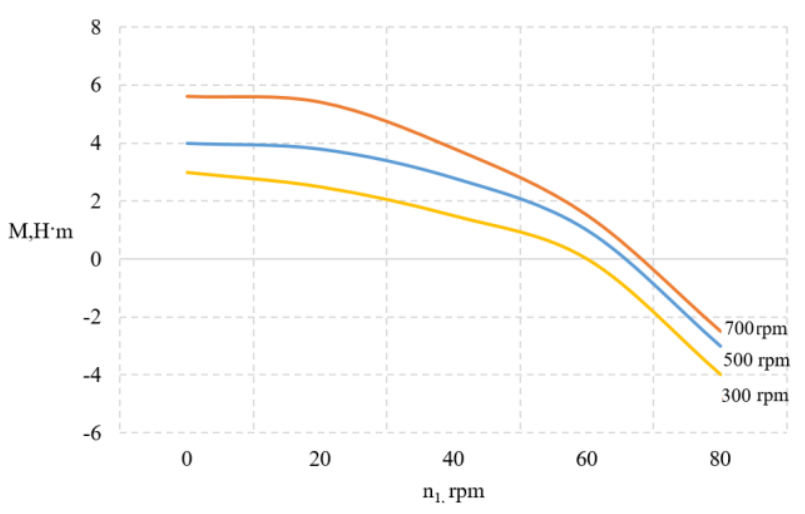

a

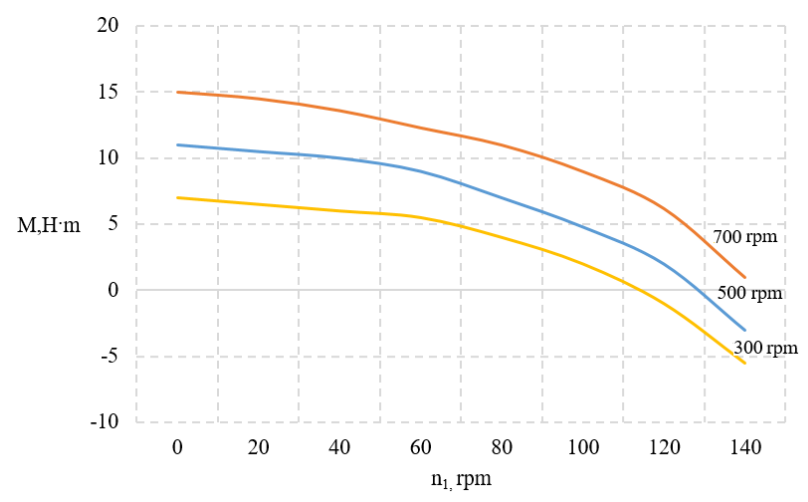

$\mathrm{c}$

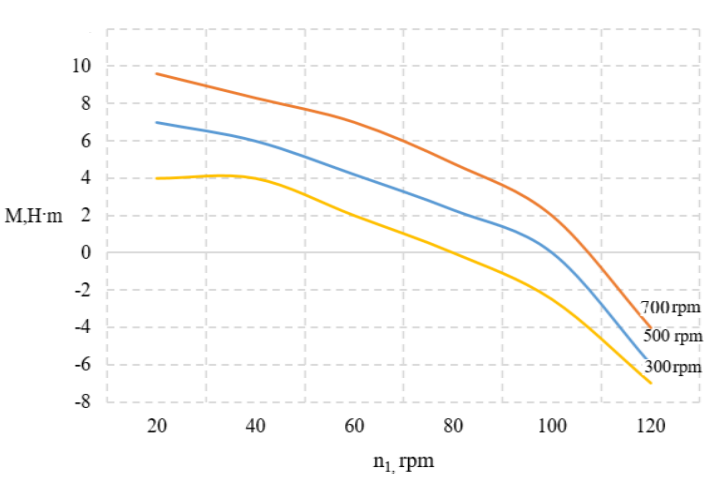

B

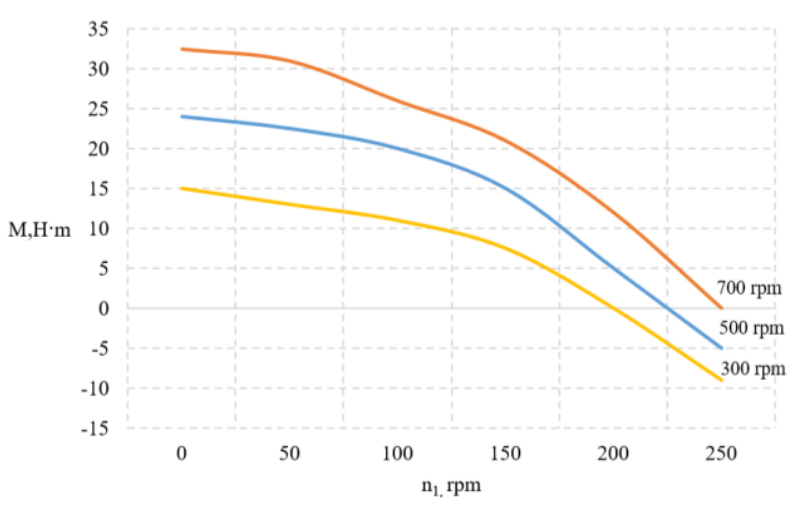

d

Fig.4. Dependence of the moment of forces on the speed of free rotation of the wind wheel at: a) $\mathrm{v}=3 \mathrm{~m} / \mathrm{s}$; b) $\mathrm{v}=5 \mathrm{~m} / \mathrm{s}$; c) $\mathrm{v}=7 \mathrm{~m} / \mathrm{s} ; \mathrm{d}) \mathrm{v}=15 \mathrm{~m} / \mathrm{s}$;

As you can see, the results obtained with a sufficient degree of accuracy $\left(R^{2}>0.9972\right)$ are approximated by dependences of the form:

$$
n_{1}(n, v)=A(n) v^{B(n)} \text {. }
$$


In this formula the free stream velocity is given in $\mathrm{m} / \mathrm{s}$, and the rotation speeds of the cylinder's $\mathrm{n}$ and $\mathrm{n}_{1}$ in rpm. It can be shown that the functions $A(n)$ и $B(n)$ and have the following form: $A(n)=12.958 n^{0.1224}$, $B(n)=0.4483 n^{0.0881}$.

\section{Conclusions}

A mathematical model with the computational domain of the wind wheel has been developed using the ANSYS program. A picture of an air flow with a three-dimensional vortex characteristic of a turbulent flow at high Reynolds numbers is obtained. The dependences of the influence of the rotation speed of the wind turbine on the moment of forces acting on the wind wheel are numerically determined at various speeds of the incoming air flow and different speeds of rotation of the cylinders. It has been established that the magnitude of the wind wheel the moment of forces increases in direct proportion to the revolutions number of rotating cylinder and the angular velocity of the wind wheel. It should be noted that the results obtained are valid within the framework of given mathematical model with the accepted assumptions and boundary conditions.

\section{REFERENCES}

1 Shedlovskii I. A. Experimental study of a multi-blade wind wheel model. Tekhn. Mekhanika. 2017, No. 2, pp. $61-72$. [in Russian]

2 Bychkov N.M., Dovgal A.V., Kozlov V.V. Magnus wind turbines as an alternative to the blade ones. Journal of Physics: Conference Series. 2007, Vol. 75, pp. 012004.

3 Isaev S.A., Baranov P.A., Kudriavtsev N.A., Zhukova Iu.V. Numerical simulation of unsteady heat exchange at a turbulent flow around a circular cylinder. Part 1. Methodic study. Thermophysics and Aeromechanics. 2005, Vol.12, No.1, pp. 27-38.

4 Marzuki O.F., Mohd Rafie A.S., Romli F.I., et al. An overview of horizontal-axis Magnus wind turbines. AEROTECH VII - Sustainability in Aerospace Engineering and Technology. Proceeding of the IOP Conf. Series: Materials Science and Engineering. 2018, Vol. 405, pp. 012011.

5 Bobkov V. G., Bondarev A.E., Zhukov V. T., Manukovskii K.V., Novikova N.D., Feodoritova O. B. Numerical simulation of dynamics of vertical-axis wind turbines. Preprint. The Keldysh Institute of Applied Mathematics of the RAS. 2019, No. 119, 25 p. doi:10.20948/prepr-2019-119 [in Russian]

6 Obukhov S.G. A method for modeling the mechanical characteristics of low-power wind turbines. Alternative energy and ecology. 2011, No. 1(93), pp. $12-17$.

7 Lee K.-J., Yang H.-D., Park S.-H., et al. Characteristics of Mechanical and Electrical Power Transmission for Small-Scaled Wind Turbine. World Journal of Engineering and Technology. 2016, Vol.4, No. 3D, October 2016. DOI: $10.4236 /$ wjet.2016.43D011 .

8 Singh M., Santoso S. Dynamic Models for Wind Turbines and Wind Power Plants. National Renewable Energy Laboratory. Texas, University of Texas at Austin, 2011, 115 p.

9 ANSYS Fluent software. Available at: www.ansys.com/products/fluids/ansys-fluent

10 Computer modeling of 3D-models of aviation equipment and engineering calculations. Available at: www.ipm ce.ru/custom/vsop/themes/3dmodel/

11 Kussaiynov K., Sakipova S.E., Tanasheva N.K., Kambarova Zh.T., et al. Wind turbine based on the Magnus effect. Innovative patent No. 30462. Publ. 23.09.2015, 7 p.

12 Sakipova S.E., Tanasheva N.K., Minkov L.L. Modeling aerodynamics of a wind turbine with cylindrical blades in a turbulent air flow. Eurasian Physical Technical Journal. Karaganda, 2020, Vol.17, No. 1(33), pp. 106 -112.

13 Moon J.S., Manuel L. Toward understanding waked flow fields behind a wind turbine using proper orthogonal decomposition. Journal of Renewable and Sustainable Energy. 2012, Vol. 13, pp. 023302; doi.org/10.1063/5.0035751 